\title{
Nitration Studies in Some $\beta$-Carboline Bases
}

\author{
Salimuzzaman Siddiqui, Viqar U. Ahmad, M. Ataullah Khan, \\ and SaIra Ismail \\ Postgraduate Institute of Chemistry, University of Karachi, \\ Karachi, Pakistan \\ (Z. Naturforsch. 28 b, 486-487 [1973]; received December 19, 1972)
}

Nitration, $\beta$-carboline bases, NMR spectroscopy

Chemical and pharmacological studies of the nitration products of some $\beta$-carboline bases are presented. The position of the nitro group in the products has been established mainly by NMR spectroscopy.

In the course of studies on the correlation of structure and antiaryhthmic cardiac action in the ajmaline series of bases, mononitroajmaline was prepared $^{1}$ by nitration of diacetylajmaline in glacial acetic acid medium and subsequent hydrolysis.

With the help of NMR spectrum of the nitro base in $\mathrm{CF}_{3} \mathrm{COOH}$, it was possible to assign the position of the nitro group at $\mathrm{C}-10$.

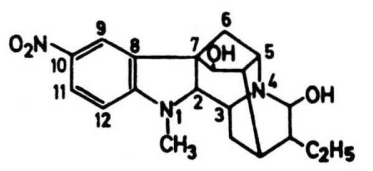

10-Nitroajmaline

In the aromatic region of the spectrum, a three spin system is present. There is a signal at $3.01 \tau$ due to $\mathrm{H}^{-12}$ which is split into a doublet $(J=9 \mathrm{cps}$, ortho coupling with $\mathrm{H}^{-11}$ ). It does not show any meta coupling but only a small para coupling of $0.6 \mathrm{cps}$. The signal of $\mathrm{H}^{-11}$ is a quartet centered at $1.65 \tau$ and shows, in addition to the ortho coupling with $\mathrm{H}^{-12}$, a meta coupling with $\mathrm{H}^{-9}(J=2.5 \mathrm{cps})$. There is a doublet at $1.5 \tau$ due to $\mathrm{H}^{-9}$ indicating a meta coupling with $\mathrm{H}^{-11}$ of 2.5 cps.

The pharmacological investigation of the antiarythmic activity of nitroajmaline revealed that it is more than twice as active as ajmaline ${ }^{2}$.

Profitting from these findings, it appeared of interest to extend the nitration studies to some other $\beta$-carboline bases. Of particular interest in this connection was the introduction of a nitro group in reserpine in order to note any changes in its dual hypotensive and sedative central depressant action.

Requests for reprints should be sent to Dr. V. U. Aнmad, Post Graduate Institute of Chemistry, University of Karachi, Karachi-32, Pakistan.
As a result of considerable experimentation, it was ultimately possible to arrive at the optimum experimental conditions for the reaction. When carrying out the nitration of reserpine in glacial acetic acid at $18-20^{\circ} \mathrm{C}$ with the reaction period of about 4-6 $\mathrm{min}$, three position isomers of nitroreserpine could be obtained, all of which analysed well for $\mathrm{C}_{33} \mathrm{H}_{39} \mathrm{O}_{11} \mathrm{~N}_{3}$. The major product (m.p. $146{ }^{\circ} \mathrm{C}$ yield $45 \%$ ) could be characterised as 1-nitroreserpine mainly by spectral studies. It yielded 3,4,5trimothoxybenzoic acid and a nitroamino acid on alkaline hydrolysis. There was no peak due to indole $\mathrm{NH}$ in the IR and NMR spectra of the nitro base as well as those of the nitroamino acid indicating a substutition at indole nitrogen. The NMR spectra of the two compounds showed signals for five and three protons respectively in the aromatic region. The $\mathrm{H}^{-12}$ signal is shifted downfield while $\mathrm{H}^{-3}$ is shifted upfield by $0.3 \mathrm{ppm}$ compared with reserpine.

The position of the nitro group was further confirmed by the reduction of l-nitroreserpine by zinc and dilute hydrochloric whereby the nitro group was removed and the nitro base was converted back into reserpine.

The second product (m.p. $231^{\circ} \mathrm{C}$ yield $10-12 \%$ ) showed in the NMR spectrum $\left(\mathrm{CDCl}_{3}\right)$ two signals at $2.35 \tau$ and $3.10 \tau$ due to $\mathrm{H}^{-9}$ and $\mathrm{H}^{-10}$ showing the characteristic ortho coupling of $J=8.5 \mathrm{cps}$ indicating that the nitro group is located at $\mathrm{C}^{-12}$. It yielded 3,4,5-trimethoxybenzonic acid and a nitroamino acid on alkaline hydrolysis. The third, carmine coloured, isomer, m. p. $253^{\circ} \mathrm{C}$ (decomp.) which was obtained in a very small yield, appears to be 9nitroreserpine although its structure could not be settled finally due to the paucity of the material.

Pharmacological tests of l-nitroreserpine have 


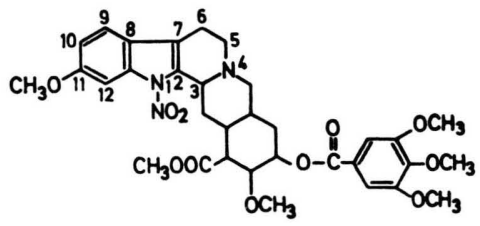

1-Nitroreserpine

shown that when administered intravenously its hypotensive activity is of the same order as that of reserpine, with only mild sedative properties, and none of its undesirable side effects. 12-Nitroreserpine requires higher doses to produce the same hypontensive activity as reserpine but its action is much longer lasting.

12-Nitroreserpine, when reduced with iron and glacial acetic acid in the hot, yielded a base which did not crystallise. It was acetylated with acetic anhydride in pyridine whereby 12 -acetamidoreserpine was obtained as a white crystalline solid, m. p. 250-56 ${ }^{\circ} \mathrm{C}$ (decomp.).

The nitration reaction was subsequently followed up with two more $\beta$-carboline bases, harmine and harmidine $\mathrm{e}^{3}$. The nitration of harmaline, which has recently been shown ${ }^{4}$ to be a mixture of harmine and harmidine, was carried out by FRITZSCHE ${ }^{5}$ and later by FISCHER and BOESLER ${ }^{6}$. The latter authors obtained nitroharmaline, m.p. $122^{\circ} \mathrm{C}$, along with nitroapoharmine carboxylic acid and $m$-nitroanisic acid by reacting the base with nitric acid $(d 1.15)$ at room temperature. The nitration reaction was subsequently carried out by Konovalova et al. ${ }^{7}$ who also obtained mononitroharmaline, m.p. $132{ }^{\circ} \mathrm{C}$ (decomp.), which could be oxidised to mononitroharmine, m.p. $204-205^{\circ} \mathrm{C}$. The position of the nitro group was assumed by these authors to be para to indole nitrogen (C-6). On carrying out the nitration reaction with harmidine in glacial acetic acid medium, it was possible to obtain a mononitro derivative, m.p. $120-22{ }^{\circ} \mathrm{C}$, in almost theoretical yield. The NMR spectrum $\left(\mathrm{CDCl}_{3}\right)$ clearly reveals that the nitro group is present at $\mathrm{C}^{-8}$. In the aromatic part of the spectrum there are two doublets at $2.22 \tau$ and $3.07 \tau$ due to $\mathrm{H}^{-6}$ and $\mathrm{H}^{-5}$ respectively showing an ortho coupling constant of $8.5 \mathrm{cps}$.

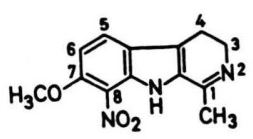

8-Nitroharmidine

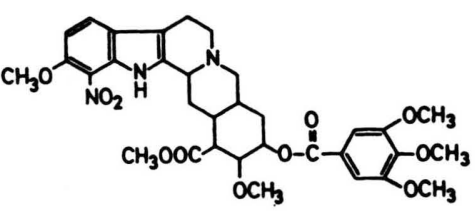

\section{2-Nitroreserpine}

The nitration of harmine, however, appeared to proceed in a very complicated manner. After considerable experimentation, it was possible to obtain mononitroharmine, $\mathrm{C}_{13} \mathrm{H}_{11} \mathrm{~N}_{3} \mathrm{O}_{3}$, by treatment of the base with conc. $\mathrm{HNO}_{3}$, at $15-20^{\circ} \mathrm{C}$ (yield $55 \%$ ) which charred at about $260^{\circ} \mathrm{C}$ and thus appeared to be different from the nitroharmine reported by Fischer and BOESLER ${ }^{6}$. The same nitro derivative could be obtained by carrying out the reaction in glacial acetic medium or treatment of harmine nitrate with conc. $\mathrm{H}_{2} \mathrm{SO}_{4}$. The position of the nitro group in nitroharmine was fixed at $\mathrm{C}^{-6}$ from NMR spectrum. In the aromatic region, there are 3 groups of signals. The signal of $2.90 \tau$ can be assigned to $\mathrm{H}^{-8}$ due to its position. It shows a small splitting of $0.2 \mathrm{cps}$ due to para coupling with $\mathrm{H}^{-5}$. The signal of $\mathrm{H}^{-5}$ is at $1.28 \tau$ with the same coupling constant. $\mathrm{H}^{-3}$ and $\mathrm{H}^{-4}$ show a typical $\mathrm{AB}$ spectrum; there is a complex multiplet of peaks at about $1.95 \tau$ equivalent to two protons showing a coupling constant of $6 \mathrm{cps}$.

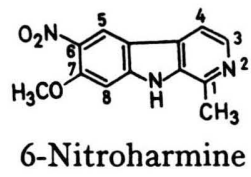

Further work on the nitration of other carboline bases and pharmacological investigation of the products is in progress.

1 S. Siddiqui and V. U. Ahmad, Proc. Pak. Acad. Sci. 5, 1 [1968].

2 Private Communication from F. Merck, Darmstadt, W. Germany.

3 S. Siddiqui, Pak. J. Sci. Ind. Res. 5, 207 [1962].

4 S. Siddigui and R. Kamal, Pak. J. Sci. Ind. Res. 7, 1 [1964].

5 J. Fritzsche, Liebigs Ann. Chem. 68, 355 [1848]; 88, 329 [1853].

B O. Fischer and W. Boesler, Chem. Ber. 45, 1930 [1912].

7 R. Konovalova and A. Orekhov, Arch. Pharm. 273, 156 [1935]. 